\title{
REVIEW
}

\section{Where now for opioids in chronic pain?}

\author{
Dr Cathy Stannard \\ Consultant in Complex Pain and Pain Transformation Programme Clinical Lead, NHS Gloucestershire CCG, \\ Sanger House, 5220 Valiant Court, Gloucester Business Park, Brockworth, GL3 4FE \\ Correspondence to Dr Cathy Stannard, cfstannardaaol.com
}

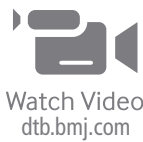

Key learning points

- Opioids are valuable in the management of acute pain, pain related to cancer and for pain management at the end of life.

- There is a lack of robust evidence on the benefit of long-term opioids in the management of chronic pain.

- Inappropriate use of long-term opioids in chronic pain is associated with serious adverse effects.

- The risk of harm from opioids increases significantly above a dose equivalent to $120 \mathrm{mg} / \mathrm{day}$ of oral morphine.

- In conjunction with the patient, regularly review the effect of opioid treatment and consider whether there is a need to reduce the dose or stop the opioid.

\section{Introduction}

Chronic pain has always been and will remain difficult to manage. There have been important developments in our understanding of the neurobiology of pain, but perhaps the greatest advance is our ability to analyse trial data more critically and to realise that we may have been wrongly optimistic about the use of some therapies. Although the publication of high-quality literature reviews may have reduced our therapeutic options, it also provides an opportunity to improve patient care by ensuring that we are not exposing patients with pain, whose lives are difficult enough as it is, to the harms of medicines that do not help them.

In particular, there is a need to reconsider the place of opioids in the management of chronic pain. This includes the so-called weak opioids (eg, codeine and dihydrocodeine), and strong opioids (eg, morphine, oxycodone, fentanyl and buprenorphine), as well as tramadol (a non-selective agonist at mu, delta and kappa opioid receptors with monoaminergic properties), which should be considered alongside the strong opioids.

Opioids emerged into the chronic pain management scene in the 1990s. The recognition of their potent role in the management of acute and end-of-life pain, the inappropriate adoption of WHO analgesic ladder designed for use in cancer pain at the end of life to the management of chronic pain, the observably refractory nature of persistent pain, and the influence of marketing by the pharmaceutical industry fuelled an increase in the popularity of opioids as a treatment for chronic pain. Early trials, some randomised and controlled, seemed to provide sufficient evidence to support this approach.
In 2013, it became apparent that the rise in the prescribing of opioids was accompanied by a parallel rise in opioid-related harms, including addiction, overdose and death. This prompted a reappraisal of the early clinical trials, and their short duration, constrained dosing and inability to capture evidence on longer term harms suggested that opioid use in clinical practice was neither as safe nor effective as we had previously thought. ${ }^{1,2}$ The opioid epidemic in North America grew into a public health catastrophe, and scrutiny of the clinical effectiveness and harms of opioids has intensified. The picture in Europe is nuanced by a number of cultural and healthcare structural influences, and currently the scale of opioid misuse and mortality remains small compared with the USA. ${ }^{3}$ However, opioid prescribing continues to increase despite clinical evidence that this is unlikely to be a helpful strategy. The problem does not seem to be awareness of the evidence, but rather hopelessness about what to do instead. ${ }^{4}$

\section{Opioids and evidence}

There are a large number of systematic reviews, and more recently reviews of systematic reviews of opioid therapy for chronic pain. ${ }^{5-9}$ Evidence on the benefits of long-term opioid therapy is lacking. There is no evidence that any opioid is more effective than others and little evidence that opioids differ in their propensity to cause harm. An obvious limitation is the short duration of many clinical trials. A systematic review of open-label extensions of clinical trials gives some insight into opioid use up to a year and suggests that some patients can achieve sustained benefit from opioids. However, patients in this review were those selected for clinical trials who were monitored 
and supervised closely, and the authors comment that firm conclusions cannot be drawn about long-term use in clinical practice. $^{10}$

Data on opioid harms come from clinical trials and large cohort studies that mostly involved strong opioids. Common adverse effects include dizziness, somnolence, nausea and constipation. ${ }^{8}$ Longer term harms include fractures, falls, endocrine and immune disturbance, disturbance of nocturnal respiratory control, and consequent cardiovascular adverse events. ${ }^{11-16}$ The prevalence of addiction to prescription opioids is variably reported and may be context-specific. One systematic review suggested that addiction occurs in $8 \%-12 \%$ of individuals taking prescription opioids, but most data in the review derive from the USA and we have no definitive figures for European populations. ${ }^{17}$

The likelihood of harms from initiation of opioid treatment relates to the duration of treatment, dose and formulation. Short-term or intermittent opioid therapy is safer than continuous prescribing, particularly at high doses. The precise dose at which harms outweigh benefits is difficult to determine accurately, and the nature of the risks is somewhat culturally and contextually specific. In the UK, the Faculty of Pain Medicine has advised that above a dose equivalent to $120 \mathrm{mg} /$ day of oral morphine, the risk of harm increases substantially with no additional benefit, ${ }^{18}$ although this is only a guide, and suggested thresholds for prescribing vary from country to country. However, this figure does not imply that lower doses are always safe, but rather emphasises the message that dose escalation in the face of poor pain relief is an inappropriate strategy for treatment of long-term pain. There is also evidence that use of prolonged-release opioids is more problematic than use of immediaterelease preparations. Immediate-release opioids allow flexibility in dosing and patients can be encouraged to look for opportunities to avoid taking opioid analgesia, using the medicine only when pain is more severe than usual and to support achievement of specific functional goals. Use of medicines intermittently is less likely to be associated with tolerance, and patients describe a more noticeable effect if using immediate-release medicines periodically. ${ }^{18-21}$ The nature and periodicity of patients' pain will ultimately inform the choice of regimen, but schemes that include at least part of the daily opioid load given as an immediate-release preparation are safer and more effective.

\section{Risk mitigation: minimising opioid-related harms $^{18,19,22-24}$}

Who might benefit from opioids?

Opioids should not be prescribed on the basis of reported intensity of symptoms. The severity of underlying pathology makes little contribution to the intensity of pain experienced and articulated by means of a 0-10 scale. More important influences on the reported pain intensity are pain, anxiety, concern about the meaning and impact of the pain and non-pain-related worries, and current and past emotional burdens. ${ }^{25}$

There is no particular type of persistent pain that is more suitable for or responsive to opioid treatment. Neuropathic pain was previously described as opioidresistant, but evidence suggests that opioids are no less helpful for neuropathic pain than other conditions. Holistic assessment of the person with pain underpins opioid prescribing decisions. For patients who have a readily demonstrable painful pathology, opioids are unlikely to be a long-term solution, but a trial of therapy might be considered (see below). Patients who have high levels of distress and disability, who remain in severe pain despite a number of medical (including pharmacological) interventions, and in whom an obvious ongoing painful pathological process is not evident will need detailed assessment and collaborative treatment planning, including evaluation and management of comorbid mental health problems such as anxiety and depression. Opioids should not be prescribed to provide temporary alleviation of distress. ${ }^{26}$

Who might be harmed from opioids?

At particular risk are patients with sleep apnoea in whom opioids are contraindicated, unless the patient fully adheres to treatment for apnoea, in which case a cautious closely supervised trial of opioids might be considered in collaboration with the patient's respiratory team. Drug choice and dosing will need to be carefully considered in patients with hepatic and renal dysfunction, and usual cautions apply for prescribing in older people. Patients who are at the most risk of developing problematic opioid use (including addiction) are those prescribed opioids more frequently, in higher doses and in combination with other centrally acting drugs, particularly benzodiazepines. This phenomenon is described as adverse selection. ${ }^{27}$ Patients who have comorbid mental health diagnoses and have a history of substance misuse, particularly opioid misuse, are more at risk.

\section{Trying out opioids}

Given the accumulating evidence of harm associated with opioids and the poor evidence for efficacy, it is not surprising that there is increasing reluctance to prescribe opioids for chronic pain. However, other drug treatments and non-pharmacological interventions are probably similarly ineffective and have their own adverse effects, so should not be offered simply to avoid prescribing opioids. $^{28}$

A small number of patients may derive medium-term to long-term benefit from pain medicines, including opioids: if a patient achieves $>50 \%$ pain reduction, this 
- Opioid medicines are powerful painkillers but work best for short-term pain. They are less helpful for long-term pain.

- A small proportion of people with long-term pain may get benefit from opioids, and if you and your prescriber decide to try this out you should discuss a trial of treatment carefully.

- There are no medicines or other treatments that work well for long-term pain, so your healthcare team will work with you to make the impact of pain on your life as manageable as possible.

- If you are on high doses of opioids for long-term pain, this puts your health at significant risk. You should discuss with your prescriber a plan for slowly reducing the dose.

- Tell your healthcare team about your life, other problems you experience and the impact that long-term pain has on you so that they can support you in as many ways as possible.

can be accompanied by improvement in mobility, sleep and other functional domains. ${ }^{23}$ For carefully selected patients, therefore, a carefully managed short trial of opioid treatment is a reasonable choice to show whether pain reduction is achievable for that patient. ${ }^{11}$ Short-term efficacy does not predict long-term efficacy, but patients who do not achieve useful pain reduction from a short (2 weeks) trial will not benefit from extended prescribing and opioids need not be further explored. However, establishing the regimen for longer term prescribing is not the purpose of the trial. A structured approach to the opioid trial is helpful.

1. Discuss the desired outcome of the trial. This will usually be pain reduction, but this should be assessed by agreeing a goal, currently not possible because of pain, that might be achieved if useful pain relief occurs. Goals might include completion of a modest activity or improved sleep.

2. Agree how long the trial will last. If a patient has continuous pain, 1 week will be sufficient to assess whether opioids might be helpful. If the patient has intermittent pain or flare-ups of pain for which they would like to try additional analgesia, then the trial can be extended to allow the patient to try opioids on two or three occasions when the pain is severe.

3. Immediate-release oral morphine is the best option to assess response to opioid treatment. The patient may need to try different doses within a specified range. Suggest starting with a dose of 5-10 mg for an episode of severe pain. If a single dose of $20 \mathrm{mg}$ morphine does not reduce pain usefully, long-term prescribing is not reasonable. A record of opioid use with comment on activity, sleep and anything that may have affected the pain during the trial is helpful for assessment of the outcome.

4. If the trial is unsuccessful, the opioid prescription can be tapered and stopped within 1 week.

5. Document details of the opioid trial. If the trial is successful, it is useful to have the rationale for longer term prescribing on record. Similarly, a welldocumented unsuccessful trial helps future prescribers make informed choices about prescribing.

Medium-term to long-term prescribing

The details of a successful opioid trial help determine the regimen for longer term prescribing. This should reflect the characteristics of the patient's pain and needs close monitoring to minimise adverse effects and ensure continued efficacy. One or two small upwards adjustment in the opioid dose may be reasonable during the titration phase of the longer term prescription, but if the agreed dose based on trial response makes little impact on the pain it is unlikely that opioids are going to be successful in the longer term.

\section{Risk mitigation: managing established opioid problems}

Regular review of all medicines is important to determine that they are still needed and effective. Patients who are on high-dose opioids (>120 mg morphine equivalent daily) and those on multiple opioids and opioids in combination with benzodiazepines, gamma-aminobutyric acid analogues (gabapentin and pregabalin) and other drugs such as hypnotics are at risk of serious adverse events, particularly overdose and cardiorespiratory complications. If patients describe continuing severe pain despite opioids, the treatment is ineffective and the patient should be encouraged and supported to taper and stop the medicines. Stopping medicines that are ineffective is an important healthcare intervention: if the opioids are poorly effective, the dose taper is usually not associated with increased pain, and patients are better able to manage their symptoms when free from opioid adverse effects. If opioid treatment is effective only at doses thought to be unsafe, opioids are not a good option for that patient and she/he needs to be supported to reduce the dose. Dose reduction should never be abrupt and the plan should be agreed with the patient and other professionals involved in the patient's pain 
management. Reduction of opioids by $10 \%$ of the total dose per fortnight usually avoids withdrawal symptoms (the dose decrement decreases as the baseline dose becomes lower). For patients who have been on high-dose opioids for a long time, reasonable goals should be set, and if good progress is being made with dose reduction it is reasonable to pause the taper intermittently while the patient adjusts to the lower dose. All dose adjustments should be preceded by detailed evaluation so that the patient can be supported in other ways to manage their pain, including help in becoming more mobile, help with sleep, addressing social isolation and managing comorbid emotional problems.

If patients are reluctant to engage with dose reduction, this may be because they are fearful of worsening pain or inability to get through the day without medicines. These individuals and those on very high doses of opioids will need support from a multidisciplinary team, including general practitioners and practice pharmacists and specialists in addiction and pain medicine. Occasionally, inpatient detoxification is the only safe option, but such treatment is extremely difficult to access, and drug and alcohol addiction facilities may be a poor context in which to manage patients with pain and problematic prescription opioid use.

\section{The future}

Effective drug therapies for long-term pain are unlikely to emerge and a shared recognition by healthcare professionals and people with pain that medicines play only a small role in pain management will help to reduce harms. The goal of long-term pain management is to support the patient in living as full a life as possible despite ongoing pain symptoms and to ensure that optimum attention is given to emotional and social difficulties. Opioids are, however, an option to support some patients in managing symptoms, and they may be prescribed with caution if they are effective in low doses and used intermittently as part of a broader pain management plan. There is, however, a significant population of patients who remain on medicines that provide little benefit and cause harm. We are now dealing with the early enthusiasm for opioid prescribing and are seeing emerging problems with gabapentin and pregabalin. ${ }^{29}$ We should learn from this and try to gain a better understanding of the influences on prescriber behaviour (particularly when there are few alternatives) and potentially complex antecedents that lead to a desire to use medicines.

\section{Competing interests None declared.}

Provenance and peer review Commissioned; externally peer reviewed.

(C) Author(s) (or their employer(s)) 2018. No commercial re-use. See rights and permissions. Published by BMJ.

\section{References}

1 Ballantyne J, Mao J. Opioid therapy for chronic pain. N EnglJ Med 2003; 349: 1943-53.

2 Kissin I. Long-term opioid treatment of chronic nonmalignant pain: unproven efficacy and neglected safety? Journal of Pain Research 2013; 6: 513-29.

3 Weisberg D, Becker WC, Fiellin DA et al. Prescription opioid misuse in the USA and UK: cautionary lessons. Int J Drug Policy 2014; 25: 1124-30.

4 McCrorie C, Closs SJ, House A et al. Understanding long-term opioid prescribing for non-cancer pain in primary care: a qualitative study. BMC Fam Pract 2015; 16: 121.

5 Chou R, Turner JA, Devine EB et al. The effectiveness and risks of long-term opioid therapy for chronic pain: a systematic review for a National Institutes of Health Pathways to Prevention Workshop. Ann Intern Med 2015; 162: $276-86$.

6 Els C, Jackson TD, Kunyk D et al. Adverse events associated with medium- and long-term use of opioids for chronic non-cancer pain: an overview of Cochrane Reviews. Cochrane Database of Syst Rev 2017; 10: CD012509. DOI:10.1002/14651858.CD012509.pub2.

7 Furlan AD, Sandoval JA, Mailis-Gagnon A et al. Opioids for chronic non-cancer pain: a meta-analysis of effectiveness and side effects. CMAJ 2006; 174: 1589-94.

8 Kalso E, Edwards JE, Moore RA et al. Opioids in chronic noncancer pain: systematic review of efficacy and safety. Pain 2004; 112: 372-80.

9 Noble M, Treadwell JR, Tregear SJ et al. Long-term opioid management for chronic noncancer pain. Cochrane Database of Syst Rev 2010; 1: CD006605. DOI:10.1002/14651858.CD006605.pub2.

10 Hauser W, Bernardy K, Maier C et al. Long-term opioid therapy in chronic noncancer pain. A systematic review and meta-analysis of efficacy, tolerability and safety in open-label extension trials with study duration of at least 26 weeks. Schmerz 2015; 29: 96-108.

11 Brack A, Rittner HL, Stein C et al. Immunosuppressive effects of opioids-clinical relevance. J Neuroimmune Pharmacol 2011; 6: 490-502.

12 Correa D, Farney RJ, Chung F et al. Chronic opioid use and central sleep apnea: a review of the prevalence, mechanisms, and perioperative considerations. Anesth Analg 2015; 120: 1273-85.

13 Saunders KW, Dunn KM, Merrill JO et al. Relationship of opioid use and dosage levels to fractures in older chronic pain patients. J Gen Intern Med 2010; 25: 310-15.

14 Seyfried O, Hester J. Opioids and the Immune system. Br J Pain 2012; 6: 17-24.

15 Dunn AT, Saunders KW, Rutter CM et al. Opioid prescriptions for chronic pain and overdose. Ann Intern Med 2010; 152: 82-5.

16 Gomes T, Mamdani MM, Dhalla IA et al. Opioid dose and drug-related mortality in patients with nonmalignant pain. Arch Intern Med 2011; 171: 686-91.

17 Vowles KE, McEntee ML, Julnes PS et al. Rates of opioid misuse, abuse, and addiction in chronic pain: a systematic review and data synthesis. Pain 2015; 156: 569-76.

18 Faculty of Pain Medicine, 2015. Opioids aware: a resource for patients and healthcare professionals to support opioid prescribing for pain [online]. Available: www.rcoa.ac.uk/facultyof-pain-medicine/opioids-aware [Accessed 16 June 2018].

19 Manchikanti L, Kaye AM, Knezevic NN et al. Responsible, safe, and effective prescription of opioids for chronic 
non-cancer pain: American Society of Interventional Pain Physicians (ASIPP) guidelines. Pain Physician 2017; 20 S3-S92.

20 Miller M, Barber CW, Leatherman S et al. Prescription opioid duration of action and the risk of unintentional overdose among patients receiving opioid therapy JAMA Intern Med 2015; 175: 608-15.

21 Pedersen L, Borchgrevink PC, Riphagen II et al. Long- or short-acting opioids for chronic non-malignant pain? A qualitative systematic review. Acta Anaesthesiol Scand 2014; 58: 390-401.

22 Centers for Disease Control and Prevention, 2017. CDC guideline for prescribing opioids for chronic pain — United States, 2016 [online]. Available: https://www.cdc.gov/ mmwr/volumes/65/rr/pdfs/rr6501e1.pdf [Accessed 16 June 2018].

23 Chou R, Fanciullo G J, Fine P G et al. American Pain Society - American Academy of Pain Medicine Opioids Guidelines Panel. Clinical guidelines for the use of chronic opioid therapy in chronic noncancer pain. J Pain 2009; 10: 113-30.

24 Franklin G. Opioids for chronic non-cancer pain. A position paper of the American Academy of Neurology. Neurology 2014; 83: 1277-84.

25 Ballantyne J, Sullivan M. Intensity of chronic pain - the wrong metric? N Engl J Med 2015; 373: 2098-9.

26 Howe C, Sullivan M. The missing ' $P$ ' in pain management: how the current opioid epidemic highlights the need for psychiatric services in chronic pain care. Gen Hosp Psychiatry 2014; 36: 99-104.

27 Sullivan MD. Who gets high-dose opioid therapy for chronic noncancer pain? Pain 2010; 151: 567-568.

28 Moore A, Derry S, Eccleston C et al. Expect analgesic failure; pursue analgesic success. BMJ 2013; 346: f2690.

29 Stannard C. Misuse of gabapentin and pregabalin: a marker for a more serious malaise? Addiction 2016; 111: 1699-700.

DOI: $10.1136 / d t b .2018 .10 .000007$ 